\title{
Animal Assisted Interventions to Treat Pain and Associated Mental Health Comorbidities: A Systematized Review
}

\author{
Jacob D Graham, BS ${ }^{1}$, Matthew J Bair, MD, MS 1,2,3 \\ ${ }^{1}$ Indiana University School of Medicine ${ }^{2}$ VA Health Services Research and \\ Development Center for Health Information and Communication, Department of \\ Veterans Affairs, Veterans Health Administration. ${ }^{3}$ Regenstrief Institute, Inc.
}

\section{Background and Hypothesis:}

Pain is cited as the primary reason patients access the healthcare system and is estimated to produce total financial cost of 500 billion dollars. However, pain can be difficult to treat, and is often associated with various mental health comorbidities, such as depression, anxiety, and PTSD, which can attenuate the effects of treatment. Thus, it is important to identify novel therapies that can both provide pain relief, and treat any associated mental health comorbidity. Animal Assisted Intervention ( $\mathrm{AAl}$ ) is one such possible treatment. Numerous studies have been published that highlight the potential of AAI in this domain. The aim of this study was to evaluate and synthesize the results of these studies.

\section{Experimental Design or Project Methods:}

A literature search was conducted searching the databases MEDLINE, CINAHL, and EMBASE with search terms related to AAI, pain, anxiety, depression, and PTSD. 110 articles met the inclusion criteria and their results were reviewed

\section{Results:}

24 articles analyzed outcomes related to pain, and 17 of these articles found that AAI significantly improved pain. Of these 17 articles, the average absolute magnitude of improvement in pain severity was 15\%. 69 articles analyzed outcomes related to anxiety, and 37 found that AAI significantly improved anxiety. Of these 37 articles, the average absolute magnitude of improvement in anxiety symptom severity was $16 \% .54$ articles analyzed outcomes related to depression, and 29 found that AAI significantly improved depression. Of these 29 articles, the average absolute magnitude of improvement in depression symptom severity was $13 \%$. 15 articles analyzed outcomes related to PTSD, and 11 found that AAI significantly improved PTSD symptoms. Of these 11 articles, the average absolute magnitude of improvement in PTSD symptom severity was $18 \%$.

\section{Conclusions and Potential Impact:}

AAI is promising as a complementary therapy for the treatment of pain as it is able to significantly improve pain while simultaneously treating common mental health comorbidities. 
